of antibiotics, whether there was an explicit note in the medical chart in which the physician diagnosed a true bacteremia, and the Centers for Disease Control surveillance criteria for primary bloodstream infection. Agreement between same-strain bacteremia and each definition was examined, based on the assumption that most true infections should be the result of a single strain.

The study sample consisted of 42 patients and 106 isolates. Nineteen of the 42 bacteremias (45\%) were the same strain. Classification of bacteremias as same-strain correlated poorly with all three clinical assessments (range of percentage agreement, $50 \%-57 \%$; range of kappa statistic, 0.01-0.15). There were both false-positive and false-negative errors. Patients with three or more positive blood cultures were more likely to have same-strain bacteremia than those with only two positive cultures (11/15 [73\%] vs $8 / 27$ [30\%], $P=.006)$. Pulsed-field gel electrophoresis was more discriminating than AP PCR (percentage agreement, 83\%; kappa, 0.67).

The authors concluded that molecular typing correlated poorly with clinical criteria for true bacteremia, suggesting either that true bacteremias are frequently the result of multiple strains or that the commonly used clinical criteria are not accurate for distinguishing contamination from true bacteremia. Vancomycin treatment of clinically defined coagulase-negative staphylococcal bacteremia may frequently be unnecessary.

FROM: Seo SK, Venkataraman L, DeGirolami PC, Samore MH. Molecular typing of coagulase-negative staphylococci from blood cultures does not correlate with clinical criteria for true bacteremia. Am J Med 2000;109:697-704.

\section{Nasal Carriage as a Source of Staphylococcus aureus Bacteremia}

von Eiff and colleagues from the Institute of Medical Microbiology, Westfalische Wilhems-Universitat Munster, Munster, Germany, examined Staphylococcus aureus isolates from blood and from nasal specimens to determine whether the organisms in the bloodstream originated from the patient's own flora. In a multicenter study, swabs for culture were obtained from the anterior nares of 219 patients with $S$ aureus bacteremia. A total of 723 isolates were collected and genotyped. In a second study, 1,640 S aureus isolates from nasal swabs from 1,278 patients were collected over a period of 5 years and then compared with isolates from the blood of patients who subsequently had $S$ aureus bacteremia.

In the multicenter study of $S$ aureus bacteremia, the blood isolates were identical to those from the anterior nares in 180 of 219 patients (82.2\%). In the second study, 14 of 1,278 patients who had nasal colonization with $S$ aureus subsequently had $S$ aureus bacteremia. In $12(86 \%)$ of these 14 patients, the isolates obtained from the nares were clonally identical to the isolates obtained from blood 1 day to 14 months later.

The authors concluded that a substantial proportion of cases of $S$ aureus bacteremia appears to be of endogenous origin since they originate from colonies in the nasal mucosa. These results provide support for strategies to prevent systemic $S$ aureus infections by eliminating nasal carriage of $S$ aureus.

FROM: von Eiff C, Becker K, Machka K, Stammer H, Peters G. Nasal carriage as a source of Staphylococcus aureus bacteremia. N Engl J Med 2001;344:11-16.

\section{Granulocyte Colony-Stimulating Factor Reduces Infection Risk in Infants}

Miura and coinvestigators from Porto Alegre, Brazil, performed a randomized, double-masked, parallel-groups, placebo-controlled trial of recombinant granulocyte colonystimulating factor (rG-CSF) administration to 44 preterm neonates who had blood cultures obtained and antibiotics begun because of the clinical diagnosis of early-onset sepsis. The treatment group $(\mathrm{n}=22)$ received $10 \mu \mathrm{g} / \mathrm{kg} / \mathrm{d}$ of intravenous rG-CSF once daily for 3 days, and the placebo group $(n=22)$ received an IV preparation in the same volume.

The treatment and placebo groups were of similar gestational age ( $29 \pm 3$ vs $31 \pm 3$ weeks) and birth weight $(1,376 \pm 491$ vs $1,404 \pm 508 \mathrm{~g})$, and had similar Apgar scores and 24-hour Score for Neonatal Acute Physiology scores. The mortality rate was not different between treatment and placebo groups. However, the occurrence of a subsequent nosocomial infection was lower in the rG-CSF recipients. The rG-CSF treatment did not alter the serum concentrations of the cytokines measured (except for G-CSF). Serum G-CSF levels and blood neutrophil counts were higher in the treatment than in the placebo group 24 hours and 48 hours after dosing.

The authors concluded that the administration of three daily doses of $\mathrm{rG}$-CSF to premature neonates with the clinical diagnosis of early-onset sepsis did not improve mortality but was associated with acquiring fewer nosocomial infections over the subsequent 2 weeks.

FROM: Miura E, Procianoy RS, Bittar C, Miura CS, Miura MS, Mello C, et al. A randomized, double-masked, placebo-controlled trial of recombinant granulocyte colony-stimulating factor administration to preterm infants with the clinical diagnosis of early-onset sepsis. Pediatrics 2001;107:30-35.

\section{Predicting Nosocomial Infections and Deaths in ICU Patients}

Nosocomial (hospital-acquired) infections are very frequent in ICUs. The risk of death after severe infection is high, but the precise rate of death in ICU attributable to nosocomial infection is not known. Escolano and coinvestigators from Paris have reported on a project to build a statistical model to predict the occurrence of nosocomial infections in ICU and the outcome of the patients. They collected data on 676 consecutive patients admitted to an ICU for more than 24 hours between 1993 and 1996. For each patient, data were collected on history, clinical examination at entry, subsequent infections, and outcome. A multistate 
heterogeneous semi-Markov model was determined and then validated; the initial data set was randomly split into two groups: two thirds (450 patients) to build the model and one third (226 patients) to validate it. The model defined five states: ICU admission, first simple infection, first complicated infection, death, and discharge from the ICU. Transitions between these states determined nine different events. The global model of patient histories can be divided into nine survival models, each corresponding to one of these events. The possible events from a given state were considered to be competing. Since many risk factors induced nonproportional hazard functions, piecewise exponential models were used to model event occurrence. The effect of continuous covariates on hazard functions has been described with a nonparametric method that enables nonlinear relations to be shown.

Among other things, the model allows patients' postadmission histories to be predicted from data available at ICU admission. The bootstrap estimator of the attributable risk of death due to simple or complicated nosocomial infections is $44.2 \%$ (95\% confidence interval, 26.0-61.6). They were also able to characterize the most highly exposed patients, those who comprise the high-risk group on whom prevention efforts must be focused.

FROM: Escolano S, Golmard JL, Korinek AM, Mallet A. A multi-state model for evolution of intensive care unit patients: prediction of nosocomial infections and deaths. Stat Med 2000;19:3465-3482.

\section{Testing Disinfectant Efficiency on Pseudomonas Biofilm}

Wirtanen and colleagues from VTT Biotechnology and Food Research, Espoo, Finland, conducted studies on the effect of disinfectants on biofilms. Biofilms of the gram-negative bacteria Pseudomonas aeruginosa and Pseudomonas fragi were grown on stainless steel surfaces (AISI 304, 2B) for 4 days in slime broth. These biofilms were treated with four commercial disinfectants. The disinfectants were alcohol-based, tenside-based, peroxidebased, and chlorine-based products, covering most disinfectant types used in the food industry. The effects of the disinfectants on the bacterial cells were first investigated in suspension using the permeabilization test, which is based on fluorescence assessment of hydrophobic 1-Nphenylnaphtylamine. The surfaces covered with disinfectanttreated biofilms were investigated using conventional cultivation, impedimetry, and epifluorescence microscopy in combination with image analysis of preparations stained with the DNA stain acridine orange and with the metabolic indicator system CTC-DAPI.

The results showed that the tenside-based and peroxide-based disinfectants permeabilized the cells in suspension. The overall biofilm results showed that, of the agents tested, the peroxide-based and chlorine-based disinfectants acted most effectively on cells in biofilms.

FROM: Wirtanen G, Salo S, Helander IM, MattilaSandholm T. Microbiological methods for testing disinfectant efficiency on Pseudomonas biofilm. Colloids Surf $B$ Biointerfaces 2001;20:37-50.

\section{New Oligosaccharide Antibiotic for Gram-Positive Nosocomial Pathogens}

Gram-positive bacteria are increasingly found to be causative pathogens in nosocomial infections, and the occurrence of vancomycin resistance in enterococci, as well as staphylococci, has prompted Kropec and colleagues from the Institute of Environmental Medicine and Hospital Epidemiology, University Hospital Freiburg, to conduct an investigation of alternative antimicrobial agents active against these strains. Everninomycin, a new oligosaccharide antibiotic, has excellent in vitro activity against grampositive bacteria, including those resistant to vancomycin. However, avilamycin, a related compound, has been used in Europe as a growth promoter in animal food for years, and concern has been raised that cross-resistance in clinical isolates may arise.

They studied a collection of 268 nosocomial grampositive isolates from ICU patients with nosocomial pneumonia, urinary tract infection, and sepsis, using standard in vitro susceptibility testing. All species tested were sensitive to everninomycin. Furthermore, no difference could be observed between methicillin-resistant and methicillinsensitive Staphylococcus aureus or between Enterococcus faecium and Enterococcus faecalis.

These results suggest that everninomycin is a promising antibiotic for the treatment of nosocomial infections in ICU patients and that the use of a related substance as an additive in animal food has not yet promoted resistance in clinical isolates

FROM: Kropec A, Frank U, Jonas D, Thriene W, Schmidt-Eisenlohr E, Daschner FD. In vitro susceptibility to everninomycin of gram-positive nosocomial pathogens isolated from intensive care units in Germany. Chemotherapy 2001;47:15-18. 